\title{
Optimization of sensory stimulation for neuronal population activity
}

\author{
Noelia Montejo ${ }^{*}$, Jean-Luc Blanc, Yann Mahnoun, Jean-Michel Brezun, Nicolas Catz, Arnaud Norena, \\ Yohi Zennou-Azogui, Christian Xerri, Laurent Pezard \\ From Nineteenth Annual Computational Neuroscience Meeting: CNS*2010 \\ San Antonio, TX, USA. 24-30 July 2010
}

The studies of the relationship between the cortical responses and the sensory stimuli can greatly benefit from an Information Theory approach, which quantifies the information conveyed by the neuronal response about the stimulus set. Within this approach, one can select a set of stimuli called the 'optimal sensory ensemble' that maximizes the mutual information between the stimuli and the neuronal response. An adaptive algorithm $[1,2]$ and [3] allows one to solve this problem in an iterative manner.

\section{Methods}

Based on previous works, we have constructed a model of cortical network [4] in order to generalize the application of adaptive algorithm, from single cell [5] to the activity of populations of cortical neurons. The network is controlled by afferent connectivity from thalamus. This connectivity is described by a Poisson process with a rate depending on time. The procedure of off-line optimization of sensory stimuli is applied both to cortical network model and experimental data of sensory cortex (activity of single units from somatosensory cortex and multi-units from auditory cortex). In all cases, the stimulus has two control parameters: modulation and amplitude of external input for network model, frequency and locations for tactile stimulus and intensity and frequency for auditory stimulus.

\section{Results}

In the network model we obtained an optimal stimulus that corresponds to low spiking rate, which corresponds to the maximal information carried when the spontaneous activity is lightly influenced by the stimulation.
This behavior is also founded in the optimization of single unit from somatosensory cortex, where the optimal stimulus is not in receptive field of this cell. In contrast, this result is different for multi-units from auditory cortex, where the region recorded cover many receptive fields.

\section{Conclusion}

We show that the optimization procedure should be adapted to the case of multi-unit recordings to define the 'optimal sensory ensemble' based on specific parameters of the activity of populations of cortical cells. The developed algorithms can be are used to explore the process of sensory information integration in sensory cortex, and stimulation procedures aimed at improving the adaptive reorganization, for example, of maps following spinal cord lesions or auditory trauma.

Published: 20 July 2010

\section{References}

1. Arimoto S: An algorithm for computing the capacity of arbitrary discrete memoryless channels. IEEE Trans Inform Theory 18:14-20.

2. Blahut RE: Computation of channel capacity and rate distortion functions. IEEE Trans Inform Theory 18:460-473.

3. Machens CK, Gollisch T, Kolesnikova O, Herz AVM: Testing the eficiency of sensory coding with optimal stimulus ensembles. Neuron 2005, 47:447-456.

4. Wang XJ: Synaptic basis of cortical persistent activity: the importance of NMDA receptors to working memory. J Neurosci 1999, 19(21):9587-9603.

5. Machens CK: Adaptative sampling by information maximization. Phys Rev Lett 2002, 88:228107.

doi:10.1186/1471-2202-11-S1-P178

Cite this article as: Montejo et al:: Optimization of sensory stimulation for neuronal population activity. BMC Neuroscience 2010 11(Suppl 1):P178.

* Correspondence: nmmcervera@gmail.com

Laboratoire de Neurosciences Intégratives et Adaptatives UMR 6149 - CNRS -

Université de Provence, 3 Place Victor Hugo, 13331 Marseille, France 